Commun. Korean Math. Soc. 28 (2013), No. 4, pp. 669-677

http://dx.doi.org/10.4134/CKMS.2013.28.4.669

\title{
TOPOLOGICAL CONDITIONS OF NI NEAR-RINGS
}

\author{
P. Dheena and C. Jenila
}

\begin{abstract}
In this paper we introduce the notion of NI near-rings similar to the notion introduced in rings. We give topological properties of collection of strongly prime ideals in NI near-rings. We have shown that if $N$ is a NI and weakly pm near-ring, then $\operatorname{Max}(N)$ is a compact Hausdorff space. We have also shown that if $N$ is a NI near-ring, then for every $a \in N, \operatorname{cl}(D(a))=V\left(N^{*}(N)_{a}\right)=\operatorname{Supp}(a)=\operatorname{SSpec}(N) \backslash$ int $V(a)$.
\end{abstract}

\section{Introduction}

Throughout this paper, $N$ stands for a zero-symmetric near-ring with identity and all prime ideals of $N$ are assumed to be proper. We use $P(N), N^{*}(N)$ and $N(N)$ to represent the prime radical, the nilradical (i.e., the sum of all nil ideals) and the set of all nilpotent elements of $N$, respectively. An ideal $P$ of $N$ is prime if for any two ideals $A$ and $B$ of $N, A B \subseteq P$ implies $A \subseteq P$ or $B \subseteq P$. An ideal $P$ of $N$ is said to be completely prime if $a b \in P$ implies $a \in P$ or $b \in P$ for any $a, b \in N$. An ideal $S$ of $N$ is said to be completely semiprime if $a^{2} \in S$ implies $a \in S$ for any $a \in N$.

An ideal $P$ of $N$ is said to be strongly prime if $P$ is prime and $N / P$ has no non-zero nil ideals. A near-ring $N$ is said to be strongly prime if the ideal $\{0\}$ is strongly prime. An ideal $P$ of a near-ring is minimal strongly prime ideal if $P$ is minimal among strongly prime ideals of $N$. Observe that every completely prime ideal of $N$ is strongly prime and every strongly prime ideal is prime but the converses do not hold.

Note that $N^{*}(N)$ of a near-ring $N$ is the unique maximal nil ideal of $N$. For a near-ring $N, N^{*}(N)=\cap\{P \mid P$ is a strongly prime ideal of $N\}=\cap\{P \mid P$ is a minimal strongly prime ideal of $N\}$ by ([2], Lemma 1.5).

A near-ring is called reduced if it has no nonzero nilpotent elements. Now we introduce the notion of NI near-rings. A near-ring $N$ is called NI if $N^{*}(N)=$ $N(N)$. Note that $N$ is NI if and only if $N(N)$ forms an ideal if and only if

Received December 23, 2011.

2010 Mathematics Subject Classification. 16Y30, 13 C99.

Key words and phrases. NI near-ring, strongly prime ideal, weakly pm near-ring, symmetric near-ring.

This research work is supported by UGC under the major research project No. F 34$153 / 2008$ (SR). 
$N / N^{*}(N)$ is reduced. Topological properties of strongly prime ideals of NI rings have been characterized in [3]. Dheena and Sivakumar [2] have obtained only the properties of NI near-rings. Now we obtain topological properties of strongly prime ideals of NI near-rings. In this paper we study the structure of NI near-rings relating to strongly prime ideals and we associate the nearring properties and topological properties. We extend the results obtained by Hwang et al. [3] for NI rings to NI near-rings. A near-ring $N$ is called 2-primal if $P(N)=N(N)$. Clearly 2-primal near-rings are NI but the converse need not hold.

We use $\operatorname{SSpec}(N)$ and $\operatorname{Max}(N)$ for the space of all strongly prime ideals and the subspace of all maximal ideals of $N$, respectively. For any $a \in N$, we define $V(a)=\{P \in \operatorname{SSpec}(N) \mid a \in P\}$ and $D(a)=S \operatorname{Spec}(N) \backslash V(a)$. Let $V(J)=\cap_{a \in J} V(a)$, where $J$ is an ideal of $N$. Then $F=\{V(J) \mid J$ is ideal of $N\}$ is closed under finite union and arbitrary intersections, so that there is a topology on $\operatorname{SSpec}(N)$ for which $F$ is the family of closed sets. This is called the Zariski topology (see [9]). For any subset $A$ of $N,\langle A\rangle$ denotes the ideal of $N$ generated by $A$. For any $a \in N,\langle a\rangle$ stands for the ideal of $N$ generated by $a$. Note that $V(A)=V(\langle A\rangle)$ for any subset $A$ of $N$. Let $\mathscr{B}=\{D(a) \mid a \in N\}$. Then $\mathscr{B}$ is a basis for a topology of $\operatorname{SPpec}(N)$.

The operations cl and int denote the closure and the interior in $\operatorname{SSpec}(N)$. For any subset $S$ of $N$, we define $N^{*}(N)_{S}=\left\{n \in N \mid n S \subseteq N^{*}(N)\right\}$. We set $\operatorname{Supp}(a)=\cap_{x \in N^{*}(N)_{a}} V(x)$. In this paper the notations of near-ring are from [8] and the notations of topology are from [7].

\section{Preliminaries}

Following Lambek [4], we have the following definition for symmetric nearring.

Definition 2.1. A near-ring $N$ is called symmetric if $a b c=0$ implies $a c b=0$ for all $a, b, c \in N$.

Note that $N$ is symmetric if and only if $a_{1} a_{2} \cdots a_{n}=0$, with $n$ any positive integer, implies $a_{\sigma(1)} a_{\sigma(2)} \cdots a_{\sigma(n)}=0$ for any permutation $\sigma$ of the set $\{1,2, \ldots, n\}$ and $a_{i} \in N$.

We first need the following lemmas.

Lemma 2.2. For a near-ring $N$ the following conditions are equivalent:

(1) $N$ is NI.

(2) Every minimal strongly prime ideal of $N$ is completely prime.

(3) $N / N^{*}(N)$ is a subdirect product of domains.

(4) $N / N^{*}(N)$ is a reduced near-ring.

(5) $N / N^{*}(N)$ is a symmetric near-ring.

Proof. $(1) \Leftrightarrow(2)$ is proved by Dheena and Sivakumar ([2], Theorem 2.6). The other implications are straightforward. 
Lemma 2.3 ([2], Theorem 1.4). If $M$ is a multiplicative subset in $N \backslash 0$, then there exists a strongly prime ideal $P$ of $N$ such that $P \cap M=\phi$.

\section{Topological space of $S \operatorname{Spec}(N)$}

In this section, we associate the near-ring properties of $N$ and the topological properties of $\operatorname{SSpec}(N)$. A near-ring $N$ is called (weakly) pm if for each (strongly) prime ideal $P$ of $N$, there exists unique maximal ideal $M$ of $N$ such that $P \subseteq M$. Clearly pm near-rings are weakly pm near-rings. Let $A$ be a subset of $N$. We denote the lattice of all ideals of $N$ by $\operatorname{Idl}(N)$ and $S(A)=\bigcap_{A \subseteq P} P$ with $P \in \operatorname{SSpec}(N)$. $\mathbb{N}$ denotes the set of positive integers.

Lemma 3.1. Let $N$ be a near-ring and $A$ be a subset of $N$.

(1) $\operatorname{SSpec}(N)$ is a topological space with a base $\{D(a) \mid a \in N\}$.

(2) $D(A)=\bigcup_{a \in A} D(a)=D(S(A))$.

(3) $\bigcup_{i \in I} D\left(A_{i}\right)=D\left(\sum_{i \in I} A_{i}\right)$ where $A_{i}$ is a subset of $N$ containing 0 for all $i \in I$.

(4) $D(I) \cap D(J)=D(I J)$ for ideals $I, J$ in $N$.

(5) $D(I) \cap D(J)=\phi$ in $\operatorname{SSpec}(N)$ if and only if $I J \subseteq N^{*}(N)$ for ideals $I, J$ in $N$.

(6) $S(I J)=S(I) \cap S(J) \supseteq S(I) S(J)$ for $I, J \in I d l(N)$.

Proof. Straightforward.

For any subset $A$ of $S \operatorname{Spec}(N)$, we denote $\cap A=\cap_{P \in A} P$.

Lemma 3.2. Let $N$ be a near-ring. If $A$ is a subset of $\operatorname{SSpec}(N)$, then there exists an ideal $J=\cap A$ of $N$ with $\operatorname{cl}(A)=V(J)$. In particular, if $A$ is a closed subset of $\operatorname{SSpec}(N)$, then $A=V(J)$ for some ideal $J$ of $N$.

Proof. Let $P \in V(J)$ and let $D(x)$ be any arbitrary element in the basis $\mathscr{B}$ such that $P \in D(x)$. Suppose that $D(x) \cap A=\phi$. Then $x \in J$, and so $P \in V(x)$, a contradiction. Thus $D(x) \cap A \neq \phi$, and hence, the result follows from Theorem 17.5 of [7].

In view of above lemma, we have the following remark.

Remark 3.3. Let $N$ be a near-ring.

(i) The closure of $P \in \operatorname{SSpec}(N)$ is $V(P)$.

(ii) A point $P \in \operatorname{SSpec}(N)$ is closed if and only if $P \in \operatorname{Max}(N)$.

(iii) If $P, Q \in S \operatorname{Spec}(N)$ with $\operatorname{cl}(P)=\operatorname{cl}(Q)$, then $P=Q$.

With the help of Lemma 3.2, we have the following important characterizations of $\operatorname{SSpec}(N)$.

Theorem 3.4. Let $N$ be a near-ring.

(1) If $F$ is a closed set and $D(K)$ is an open set in $\operatorname{SSpec}(N)$ satisfying $F \cap \operatorname{Max}(N) \subseteq D(K)$, then $F \subseteq D(K)$.

(2) $\operatorname{SSpec}(N)$ is a compact space. 
(3) $\operatorname{Max}(N)$ is a compact $T_{1}$-space.

(4) If $\operatorname{SSpec}(N)$ is normal, then $\operatorname{Max}(N)$ is a Hausdorff space.

(5) If $N^{*}(N)=\cap \operatorname{Max}(N)$ and $\operatorname{Max}(N)$ is a Hausdorff space, then $\operatorname{SSpec}(N)$ is normal.

Proof. (1) Suppose that there is $P \in F$ with $P \notin D(K)$. Let $F=V(L)$ for some ideal $L$ of $N$. Then $K+L \subseteq P$. Hence each maximal ideal $M$ containing $P$ is also contained in $F$. Thus $M \in F \cap \operatorname{Max}(N)$, and so $M \in D(K)$, a contradiction.

(2) Let $\left\{D\left(s_{j}\right) \mid j \in J\right\}$ be an open cover of $\operatorname{SSpec}(N)$. Hence $\operatorname{SSpec}(N)=$ $\cup_{j \in J} D\left(s_{j}\right)$. Then $\phi=\cap_{j \in J}\left(\operatorname{SPpec}(N) \backslash D\left(s_{j}\right)\right)=\cap_{j \in J} V\left(s_{j}\right)=V\left(\sum_{j \in J}\left\langle s_{j}\right\rangle\right)$ which gives $\sum_{j \in J}\left\langle s_{j}\right\rangle=N$. Then there exists $K \subset J$ finite with $1=\sum_{k \in K} s_{k}^{\prime}$, where $s_{k}^{\prime} \in\left\langle s_{k}\right\rangle$ which implies $\operatorname{SSpec}(N)=\cup_{k \in K} D\left(s_{k}^{\prime}\right)$. Indeed, clearly

$$
\cup_{k \in K} D\left(s_{k}^{\prime}\right) \subseteq \operatorname{SSpec}(N)
$$

and suppose $P \in \operatorname{SSpec}(N)$ with $P \notin \cup_{k \in K} D\left(s_{k}^{\prime}\right)$. Then $s_{k}^{\prime} \in P$ for all $k \in K$ which implies $1 \in P$, a contradiction. Hence $\operatorname{SPpec}(N)$ is a compact space.

(3) For any $s_{i} \in N,\left\{D\left(s_{i}\right) \cap \operatorname{Max}(N)\right\}$ is an arbitrary open set of $\operatorname{Max}(N)$. Let $\left\{D\left(s_{i}\right) \cap \operatorname{Max}(N) \mid i \in J\right\}$ be an open cover of $\operatorname{Max}(N)$. Hence $\operatorname{Max}(N)=$ $\left(\cup_{i \in J} D\left(s_{i}\right)\right) \cap \operatorname{Max}(N)$. Then $\phi=\cap_{i \in J}\left(\operatorname{Max}(N) \backslash D\left(s_{i}\right)\right)=\left(\cap_{i \in J} V\left(s_{i}\right)\right) \cap$ $\operatorname{Max}(N)=V\left(\sum_{i \in J}\left\langle s_{i}\right\rangle\right) \cap \operatorname{Max}(N)$ which implies $\sum_{i \in J}\left\langle s_{i}\right\rangle=N$. Then there exists $J_{1} \subset J$ finite with $1=\sum_{j \in J_{1}} s_{j}^{\prime}$, where $s_{j}^{\prime} \in\left\langle s_{j}\right\rangle$, and so $\operatorname{Max}(N)=$ $\left(\cup_{j \in J_{1}} D\left(s_{j}^{\prime}\right)\right) \cap \operatorname{Max}(N)$. Therefore $\operatorname{Max}(N)$ is a compact space. Let $M_{1}$ and $M_{2}$ be two distinct elements in $\operatorname{Max}(N)$. Then $M_{1} \in D\left(M_{2}\right) \cap \operatorname{Max}(N)$ and $M_{2} \in D\left(M_{1}\right) \cap \operatorname{Max}(N)$, and so $\operatorname{Max}(N)$ is a $T_{1}$-space.

(4) Let $M_{1}$ and $M_{2}$ be distinct elements in $\operatorname{Max}(N)$. Then $\left\{M_{1}\right\}$ and $\left\{M_{2}\right\}$ are closed subsets in both $\operatorname{SSpec}(N)$ and $\operatorname{Max}(N)$. If $\operatorname{SSpec}(N)$ is normal, then there exist disjoint open sets $D(I)$ and $D(J)$ in $S \operatorname{Spec}(N)$ such that $\left\{M_{1}\right\} \subseteq D(I)$ and $\left\{M_{2}\right\} \subseteq D(J)$ for some ideals $I$ and $J$ of $N$, respectively. So $M_{1} \in D(I) \cap \operatorname{Max}(N)$ and $M_{2} \in D(J) \cap \operatorname{Max}(N)$, which imply $\operatorname{Max}(N)$ is a Hausdorff space.

(5) Let $F_{1}$ and $F_{2}$ be two disjoint closed subsets of $\operatorname{SSpec}(N)$. Then $F_{1} \cap$ $\operatorname{Max}(N)$ and $F_{2} \cap \operatorname{Max}(N)$ are also disjoint closed subsets of $\operatorname{Max}(N)$. By Theorem 32.3 in [7], $\operatorname{Max}(N)$ is normal. So there are open subsets $D(I)$ and $D(J)$ of $\operatorname{SSpec}(N)$ such that $F_{1} \cap \operatorname{Max}(N) \subseteq A, F_{2} \cap \operatorname{Max}(N) \subseteq B$ and $A \cap B=\phi$, where $A=D(I) \cap \operatorname{Max}(N)$ and $B=D(J) \cap \operatorname{Max}(N)$. Assume $N^{*}(N)=\cap \operatorname{Max}(N)$. Then $I J \subseteq \cap \operatorname{Max}(N)=N^{*}(N)$ since $D(I) \cap D(J)=$ $D(I J)$, and so $D(I) \cap D(J)=\phi$. By (1), we have $F_{1} \subseteq D(I)$ and $F_{2} \subseteq D(J)$.

Following Sun [10], we define the normality of $\operatorname{Idl}(N)$.

Definition 3.5. $\operatorname{Idl}(N)$ is called normal if for each pair $I_{1}, I_{2} \in \operatorname{Idl}(N)$ with $I_{1}+I_{2}=N$ there are $J_{1}, J_{2} \in I d l(N)$ such that $I_{1}+J_{1}=N=I_{2}+J_{2}$ and $J_{1} J_{2}=0$. 
Definition 3.6. $\operatorname{Max}(N)$ is said to be a retract of $\operatorname{SSpec}(N)$ if there is a continuous map $f: \operatorname{SSpec}(N) \rightarrow \operatorname{Max}(N)$ such that $f(M)=M$ for each $M \in \operatorname{Max}(N)$.

Lemma 3.7. Let $N$ be a near-ring.

(1) $\operatorname{SSpec}(N)$ is normal if and only if for each pair $I_{1}, I_{2} \in \operatorname{Idl}(N)$ with $I_{1}+I_{2}=N$ there are $J_{1}, J_{2} \in I d l(N)$ such that $I_{1}+J_{1}=N=I_{2}+J_{2}$ and $S\left(J_{1}\right) S\left(J_{2}\right) \subseteq N^{*}(N)$.

(2) If Idll $(N)$ is normal, then so is $\operatorname{SSpec}(N)$.

(3) If $\operatorname{Max}(N)$ is a retract of $\operatorname{SSpec}(N)$, then $N$ is a weakly pm near-ring.

(4) If $\operatorname{Idl}(N)$ is normal, then $N$ is a weakly pm near-ring.

Proof. (1) Suppose that $I_{1}, I_{2} \in I d l(N)$ with $I_{1}+I_{2}=N$ and let $F_{1}=$ $\operatorname{SSpec}(N) \backslash D\left(I_{1}\right), F_{2}=\operatorname{SSpec}(N) \backslash D\left(I_{2}\right)$. Clearly $D\left(I_{1}\right) \cup D\left(I_{2}\right)=D\left(I_{1}+I_{2}\right)=$ $D(N)=\operatorname{SSpec}(N)$, so $F_{1}$ and $F_{2}$ are disjoint closed subsets of $S \operatorname{Spec}(N)$. If $\operatorname{SSpec}(N)$ is normal, then there are disjoint open subsets $D\left(J_{1}\right)$ and $D\left(J_{2}\right)$ of $S \operatorname{Spec}(N)$ such that $F_{1} \subseteq D\left(J_{1}\right)$ and $F_{2} \subseteq D\left(J_{2}\right)$. Since $D\left(I_{1}+J_{1}\right)=$ $D\left(I_{1}\right) \cup D\left(J_{1}\right)=\operatorname{SSpec}(N)$ and $D\left(I_{2}+J_{2}\right)=\bar{D}\left(I_{2}\right) \cup D\left(J_{2}\right)=\operatorname{SSpec}(N)$, we have $I_{1}+J_{1}=N=I_{2}+J_{2}$. Since $D\left(J_{1}\right)$ and $D\left(J_{2}\right)$ are disjoint, $S\left(J_{1}\right) S\left(J_{2}\right) \subseteq$ $N^{*}(N)$. Conversely, let $F_{1}$ and $F_{2}$ be disjoint closed subsets of $\operatorname{SSpec}(N)$. Say $F_{1}=\operatorname{SSpec}(N) \backslash D\left(I_{1}\right)$ and $F_{2}=\operatorname{SSpec}(N) \backslash D\left(I_{2}\right)$. Since $F_{1}$ and $F_{2}$ are disjoint, $D\left(I_{1}\right) \cup D\left(I_{2}\right)=S \operatorname{Spec}(N)=D(N)$. By Lemma 3.1(3), $I_{1}+I_{2}=N$. Then there are $J_{1}, J_{2} \in I d l(N)$ such that $I_{1}+J_{1}=N=I_{2}+J_{2}$ and $S\left(J_{1}\right) S\left(J_{2}\right) \subseteq N^{*}(N)$. Hence $F_{1} \subseteq D\left(J_{1}\right)$ and $F_{2} \subseteq D\left(J_{2}\right)$. Clearly $D\left(J_{1}\right) \cap$ $D\left(J_{2}\right)=D\left(S\left(J_{1}\right) S\left(J_{2}\right)\right)$. By Lemma 3.1(5), $D\left(J_{1}\right)$ and $D\left(J_{2}\right)$ are disjoint.

(2) Straightforward.

(3) Suppose that $P \in \operatorname{SSpec}(N)$ and $M_{1}$ is any maximal ideal of $N$ containing $P$. Let $f: \operatorname{SSpec}(N) \rightarrow \operatorname{Max}(N)$ be a continuous retration and $f(P)=M$. Since $\{M\}$ is closed in $\operatorname{Max}(N)$, we have $f^{-1}(\{M\})$ is closed in $\operatorname{SSpec}(N)$. Since $f^{-1}(\{M\})$ contains the closure of $P, f^{-1}(\{M\})$ also contains $M_{1}$. Hence $M_{1}=f\left(M_{1}\right)=M$.

(4) Suppose that there is $P \in \operatorname{SSpec}(N)$ with $P \subseteq M_{1} \cap M_{2}$ for some distinct $M_{1}, M_{2} \in \operatorname{Max}(N)$. Since $\operatorname{Idl}(N)$ is normal and $M_{1}+M_{2}=N$, there are $J_{1}, J_{2} \in \operatorname{Idl}(N)$ such that $M_{1}+J_{1}=N=M_{2}+J_{2}$ and $J_{1} J_{2}=0$. Since $J_{1} J_{2}=0$, we have $J_{1} \subseteq P$ or $J_{2} \subseteq P$. If $J_{1} \subseteq P$ then $J_{1} \subseteq M_{1}$, a contradiction. The case of $J_{2} \subseteq P$ induces a similar contradiction.

Note that if $N$ is a NI near-ring, then $N^{*}(N)$ is completely semiprime ideal and $a b \in N^{*}(N)$ implies $\langle a\rangle\langle b\rangle \subseteq N^{*}(N)$ for any $a, b \in N$.

Combining Lemma 2.3 and Lemma 3.4, we have the following theorem.

Theorem 3.8. Let $N$ be a NI and weakly pm near-ring. Then $\operatorname{Max}(N)$ is a compact Hausdorff space. 
Proof. By Lemma 3.4(3), $\operatorname{Max}(N)$ is a compact space. Let $M_{1}, M_{2} \in \operatorname{Max}(N)$ and consider a multiplicative subset

$$
S=\left\{a_{1} b_{1} \cdots a_{n-1} b_{n-1} a_{n} b_{n} \mid a_{i} \notin M_{1}, b_{i} \notin M_{2}, i=1,2, \ldots, n, n \in \mathbb{N}\right\} .
$$

Suppose that $0 \notin S$. Then by Lemma 2.3 , there is a strongly prime ideal $P$ of $N$ with $P \cap S=\phi$ and hence $P \subseteq M_{1} \cap M_{2}$, a contradiction. So there exists $a_{i} \notin M_{1}$ and $b_{i} \notin M_{2}$ such that $a_{1} b_{1} \cdots a_{n} b_{n}=0$. Let $x_{1}=\left\langle a_{1}\right\rangle\left\langle a_{2}\right\rangle \cdots\left\langle a_{n}\right\rangle$ and $x_{2}=\left\langle b_{1}\right\rangle\left\langle b_{2}\right\rangle \cdots\left\langle b_{n}\right\rangle$ such that $x_{1} \notin M_{1}$ and $x_{2} \notin M_{2}$. Since $N$ is NI, we have $N / N^{*}(N)$ is reduced. Hence $x_{1} x_{2} \in N^{*}(N)$. Since $N^{*}(N)$ is completely semiprime, we have $\left\langle x_{1}\right\rangle\left\langle x_{2}\right\rangle \subseteq N^{*}(N)$, which implies $\left(D\left(x_{1}\right) \cap \operatorname{Max}(N)\right) \cap$ $\left(D\left(x_{2}\right) \cap \operatorname{Max}(N)\right)=\phi$ with $M_{1} \in D\left(x_{1}\right) \cap \operatorname{Max}(N)$ and $M_{2} \in D\left(x_{2}\right) \cap$ $\operatorname{Max}(N)$. Therefore $\operatorname{Max}(N)$ is a compact Hausdorff space.

We have the following corollary from Theorem 3.8.

Corollary 3.9 ([3], Lemma 3.4). If a ring $R$ is $N I$ and weakly pm, then $\operatorname{Max}(R)$ is a compact Hausdorff space.

As an immediate consequence of Theorem 3.8 or Corollary 3.9, we have the following corollary.

Corollary 3.10 ([3], Corollary 3.5). If $R$ is a 2-primal and pm ring, then $\operatorname{Max}(R)$ is a compact Hausdorff space.

Proposition 3.11. For a near-ring $N$ the following conditions are equivalent:

(1) $\operatorname{SSpec}(N)$ is normal.

(2) $\operatorname{Max}(N)$ is a retract of $\operatorname{SSpec}(N)$ and $\operatorname{Max}(N)$ is Hausdorff.

Proof. (1) $\Rightarrow(2)$ Suppose that $S \operatorname{Spec}(N)$ is normal. By Theorem 3.4(4), $\operatorname{Max}(N)$ is Hausdorff. Without loss of generality we can assume that $N^{*}(N)=$ 0 since $\operatorname{SSpec}(N)$ is canonically isomorphic to $\operatorname{S\operatorname {Spec}}\left(N / N^{*}(N)\right)$. Now for each $P \in S \operatorname{Spec}(N)$, define $F_{P}=\{I \in I d l(N) \mid I+P=N\}$. Then $F_{P}$ has the following properties: (i) if $I_{1}+I_{2} \in F_{P}$, then either $I_{1} \in F_{P}$ or $I_{2} \in F_{P}$, (ii) if $I \in F_{P}$ and $I \subseteq J$, then $J \in F_{P}$. Let $M_{P}=\sum\left\{I \in I d l(N) \mid I \notin F_{P}\right\}$. Note that $1 \notin M_{P}$ and $P \subseteq M_{P}$. Assume that $M_{P}$ is not maximal, say $M_{P} \subset M$ for some maximal ideal $M$ of $N$. Then $M \in F_{P}$ and so $M+P=N$ which implies $M=M+M_{P} \supseteq M+P=N$, a contradiction. Hence $M_{P}$ is maximal. If $P$ is maximal, then $M_{P}=P$.

Now we define a mapping $f: \operatorname{SSpec}(N) \rightarrow \operatorname{Max}(N)$ by sending each $P \in \operatorname{SSpec}(N)$ to $M_{P} \in \operatorname{Max}(N)$. Let $D(I) \cap \operatorname{Max}(N)$ be an arbitrary open subset of $\operatorname{Max}(N)$. We claim that, $f^{-1}(D(I) \cap \operatorname{Max}(N))$ is an open subset of $S \operatorname{Spec}(N)$. Let $P$ be a strongly prime ideal in $\operatorname{SPpec}(N)$ such that $P \in f^{-1}(D(I) \cap \operatorname{Max}(N))$. Then $f(P) \in D(I) \cap \operatorname{Max}(N)$. Therefore $I \nsubseteq f(P)$. Thus $I+P=N$. So there are ideals $J_{1}, J_{2}$ such that $I+J_{1}=N=P+J_{2}$ and $S\left(J_{1}\right) S\left(J_{2}\right)=0$, which implies $J_{2} \nsubseteq P$. Now we show that $D\left(J_{2}\right) \subseteq f^{-1}(D(I) \cap$ $\operatorname{Max}(N))$. Let $P_{1} \in D\left(J_{2}\right)$. Then $S\left(J_{1}\right) \subseteq P_{1}$, which gives $I+P_{1}=N$. Hence $I \in F_{P_{1}}$ and $I \nsubseteq f\left(P_{1}\right)$. Then $f$ is continuous. 
(2) $\Rightarrow(1)$ Let $g$ be a continuous retraction of $\operatorname{SSpec}(N)$ onto $\operatorname{Max}(N)$. For a closed subset $F$ of $S \operatorname{Spec}(N)$, we have $g(F)=F \cap \operatorname{Max}(N)$. If now $F_{1}$ and $F_{2}$ are disjoint closed subsets of $S \operatorname{Spec}(N)$, we can enclose $F_{1} \cap \operatorname{Max}(N)$ and $F_{2} \cap$ $\operatorname{Max}(N)$ in disjoint open sets $D(I)$ and $D(J)$ of $\operatorname{Max}(N)$, and now $g^{-1}(D(I))$ and $g^{-1}(D(J))$ are open and disjoint in $\operatorname{SSpec}(N)$ with $F_{1} \subseteq g^{-1}(D(I))$ and $F_{2} \subseteq g^{-1}(D(J))$.

Theorem 3.12. Let $N$ be a NI near-ring. Then the following conditions are equivalent:

(1) $N$ is weakly pm.

(2) $\operatorname{SSpec}(N)$ is normal.

(3) $\operatorname{Max}(N)$ is a retract of $\operatorname{SSpec}(N)$.

Proof. (3) $\Rightarrow(1)$ and (2) $\Rightarrow$ (3) follows from Lemma 3.7(3) and Proposition 3.11 .

$(1) \Rightarrow(2)$ Suppose that $N$ is weakly pm. Then $\operatorname{SSpec}(N)$ is normal by Theorem 3.8 and Proposition 3.11 when $\operatorname{Max}(N)$ is a retract of $\operatorname{SSpec}(N)$. Since $N$ is weakly pm, we can obtain a retraction $f: \operatorname{SSpec}(N) \rightarrow \operatorname{Max}(N)$ by sending each strongly prime ideal to the unique maximal ideal containing it. For a closed subset $\mathbb{F}$ of $\operatorname{Max}(N)$, we claim that $f^{-1}(\mathbb{F})$ is closed in $\operatorname{SSpec}(N)$. Let $B=\cup\{M \mid M \in \mathbb{F}\}, F=\cap\{M \mid M \in \mathbb{F}\}$ and $I=\cap\{P \in \operatorname{SSpec}(N) \mid f(P) \in$ $\mathbb{F}\}$.

Let $Q \in \operatorname{SSpec}(N)$ with $Q \subseteq B$. Then $Q+F \subseteq B$ clearly, and so there is a maximal ideal $M$ with $Q+F \subseteq M$. Thus we have $M \in \mathbb{F}$ since $\mathbb{F}$ is closed and $F \subseteq M$. Moreover, $M$ is the unique maximal ideal containing $Q$ because $N$ is weakly pm.

Now let $P \in \operatorname{SSpec}(N)$ with $I \subseteq P$. Consider any finite subset $\left\{s_{i} \mid s_{i} \notin\right.$ $B, i \leq n\}$ where $n \in \mathbb{N}$. Let $t \notin P$. Then $t \notin I$ and so there is $P_{1} \in \operatorname{SSpec}(N)$ such that $t \notin P_{1}$ and $f\left(P_{1}\right) \in \mathbb{F}$. Since $s_{i} \notin B$, we have $s_{i} \notin P_{1}$. Hence there exists $z_{i}, z_{j}^{\prime} \in N$ for $i \leq n, j \leq n-1$ such that $s_{1} z_{1} t z_{1}^{\prime} s_{2} z_{2} t z_{2}^{\prime} \cdots t z_{n-1}^{\prime} s_{n} z_{n} t \notin$ $P_{1}$. Define a multiplicative subset $X=\left\{s_{1} t_{1} s_{2} t_{2} \cdots s_{n} t_{n} \mid s_{i} \notin B, t_{i} \notin P, i \leq\right.$ $n, n \in \mathbb{N}\}$. Assume $0 \in X$ and say $s_{1} t_{1} s_{2} t_{2} \cdots s_{n} t_{n}=0$ for some $s_{i} \notin B, t_{i} \notin P$. Then there are $c_{i} \in N, i \leq n-1$ such that $t=t_{1} c_{1} t_{2} c_{2} \cdots t_{n-1} c_{n-1} t_{n} \notin P$. Hence there exists $z_{i}, z_{j}^{\prime} \in N$ for $i \leq n, j \leq n-1$ such that

$$
s_{1} z_{1} t z_{1}^{\prime} s_{2} z_{2} t z_{2}^{\prime} \cdots t z_{n-1}^{\prime} s_{n} z_{n} t \notin I .
$$

By Lemma 2.2, $N / N^{*}(N)$ is symmetric. Since $s_{1} t_{1} s_{2} t_{2} \cdots s_{n} t_{n}=0$, we have $s_{1} z_{1} t z_{1}^{\prime} s_{2} z_{2} t z_{2}^{\prime} \cdots t z_{n-1}^{\prime} s_{n} z_{n} t \in N^{*}(N)$. Thus $s_{1} z_{1} t z_{1}^{\prime} s_{2} z_{2} t z_{2}^{\prime} \cdots t z_{n-1}^{\prime} s_{n} z_{n} t \in$ $P_{1}$, a contradiction. Then there exists a strongly prime ideal $Q$ of $N$ with $Q \subseteq P \cap B$. Therefore $Q \subseteq P \subseteq M=f(P)=f(Q) \in \mathbb{F}$. Hence $\operatorname{SSpec}(N)$ is normal.

The following is an immediate corollary of Theorem 3.12.

Corollary 3.13 ([3], Theorem 3.7). Let $R$ be a NI ring. Then the following conditions are equivalent: 
(1) $R$ is weakly pm.

(2) $\operatorname{SSpec}(R)$ is normal.

(3) $\operatorname{Max}(R)$ is a retract of $\operatorname{SSpec}(R)$.

If $N$ is NI, we obtain the following results.

Theorem 3.14. Let $N$ be a NI near-ring. Then $N^{*}(N)_{S}=\cap V\left(N^{*}(N)_{S}\right)$ for any subset $S$ of $N$.

Proof. Clearly $N^{*}(N)_{S} \subseteq \cap V\left(N^{*}(N)_{S}\right)$. Let $a \in N \backslash N^{*}(N)_{S}$. Then $a S \nsubseteq$ $N^{*}(N)$. Thus as $\notin P$ for some $P \in S \operatorname{Spec}(N)$ and $s \in S$. Let $x \in N^{*}(N)_{S}$. Then $x S \subseteq N^{*}(N)$. Since $N^{*}(N)$ is completely semiprime, we have $\langle x\rangle\langle s\rangle \subseteq$ $N^{*}(N)$. Since $s \notin P$, we have $x \in P$. Then $N^{*}(N)_{S} \subseteq P$. Thus $a \notin P \in$ $V\left(N^{*}(N)_{S}\right)$ and hence $\cap V\left(N^{*}(N)_{S}\right) \subseteq N^{*}(N)_{S}$.

Lemma 3.15. Let $N$ be a NI near-ring and let $a, b \in N$. Then int $V(a) \subseteq$ int $V(b)$ if and only if $N^{*}(N)_{a} \subseteq N^{*}(N)_{b}$.

Proof. Let int $V(a) \subseteq$ int $V(b)$ for any $a, b \in N$ and let $x \in N^{*}(N)_{a}$. Then $x a \in N^{*}(N)$, and so $\langle x\rangle\langle a\rangle \subseteq N^{*}(N)$, which implies $\operatorname{SSpec}(N) \backslash V(x) \subseteq V(a)$. Then $\operatorname{SSpec}(N) \backslash V(x) \subseteq$ int $V(a) \subseteq$ int $V(b) \subseteq V(b)$, which gives $b x \in N^{*}(N)$, so $x \in N^{*}(N)_{b}$.

Conversely, let $N^{*}(N)_{a} \subseteq N^{*}(N)_{b}$ and let $P \in \operatorname{int} V(a)$. Suppose $P \notin V(b)$. Then $b \notin P$. Since $P \in \operatorname{int} V(a)$, we have $P \notin S \operatorname{Spec}(N) \backslash$ int $V(a)$. Then by Lemma 3.2, we have $\operatorname{SSpec}(N) \backslash$ int $V(a)=V(J)$ for some ideal $J$ of $N$. Since $P \notin V(J)$, we have $c \notin P$ for some $c \in J$, and so $\operatorname{SPpec}(N) \backslash$ int $V(a)=$ $V(J) \subseteq V(c)$. Clearly $a c \in N^{*}(N)$ and $b c \notin N^{*}(N)$. Then $c \in N^{*}(N)_{a}$ and $c \notin N^{*}(N)_{b}$, a contradiction. Hence int $V(a) \subseteq$ int $V(b)$.

Lemma 3.16. Let $N$ be a NI near-ring. Then for every $a \in N, \operatorname{cl}(D(a))=$ $V\left(N^{*}(N)_{a}\right)=\operatorname{Supp}(a)=\operatorname{SSpec}(N) \backslash \operatorname{int} V(a)$.

Proof. Let $P \in D(a)$ and $x \in N^{*}(N)_{a}$ for any $a \in N$. Then $a \notin P$ and $x a \in N^{*}(N)$. Since $N^{*}(N)$ is completely semiprime, we have $\langle x\rangle\langle a\rangle \subseteq N^{*}(N)$, and so $x \in P$. Thus $N^{*}(N)_{a} \subseteq P$ and hence $P \in V\left(N^{*}(N)_{a}\right)$. So $D(a) \subseteq$ $V\left(N^{*}(N)_{a}\right)$. Let $P_{1} \in \operatorname{cl}(D(a))$. Then $P_{1} \in V\left(N^{*}(N)_{a}\right)$ since $V\left(N^{*}(N)_{a}\right)$ is a closed set containing $D(a)$. Let $P \in V\left(N^{*}(N)_{a}\right)$, and let $D(x)$ be any arbitrary element in the basis $\mathscr{B}$ such that $P \in D(x)$. Suppose $P \notin D(a)$ and suppose $D(x) \cap D(a)=\phi$. Then $D(x a) \subseteq D(x) \cap D(a)=\phi$, and so $x a \in N^{*}(N)$ which implies $x \in P$, a contradiction. Thus $D(x) \cap D(a) \neq \phi$ and hence $V\left(N^{*}(N)_{a}\right) \subseteq \operatorname{cl}(D(a))$.

If $P \in D(a)$, then $P \in D(a) \cap D(x) \neq \phi$, and so $V\left(N^{*}(N)_{a}\right) \subseteq \operatorname{cl}(D(a))$. Clearly $\operatorname{Supp}(a)=V\left(N^{*}(N)_{a}\right)$. Let $P \in \operatorname{cl}(D(a))$ and suppose that $P \in$ int $V(a)$. Then there is an open set $U$ of $\operatorname{SSpec}(N)$ with $P \in U \subseteq V(a)$, and so $P \notin S \operatorname{Spec}(N) \backslash U$, a contradiction. Let $P \in S \operatorname{Spec}(N) \backslash$ int $V(a)$ and let $D(x)$ be any arbitrary element in the basis $\mathscr{B}$ such that $P \in D(x)$. Suppose that $D(x) \cap D(a)=\phi$. Then $a x \in N^{*}(N)$, and so $x \in N^{*}(N)_{a}$. But $x \notin P$, we have $N^{*}(N)_{a} \not \subset P$. Hence $P \in D\left(N^{*}(N)_{a}\right) \subseteq V(a)$, a contradiction. 


\section{References}

[1] G. F. Birkenmeier, J. Y. Kim, and J. K. Park, Regularity conditions and the simplicity of prime factor rings, J. Pure Appl. Algebra 115 (1997), no. 3, 213-230.

[2] P. Dheena and D. Sivakumar, On strongly 0-prime ideals in near-rings, Bull. Malays. Math. Sci. Soc. (2) 27 (2004), no. 1, 77-85.

[3] S. U. Hwang, Y. C. Jeon, and Y. Lee, Structure and topological conditions of NI rings, J. Algebra 302 (2006), no. 1, 186-199.

[4] J. Lambek, On the representations of modules by sheaves of factor modules, Canad. Math. Bull. 14 (1971), 359-368.

[5] G. Marks, On 2-primal Ore extensions, Comm. Algebra 29 (2001), no. 5, 2113-2123.

[6] - A taxonomy of 2-primal rings, J. Algebra 266 (2003), no. 2, 494-520.

[7] J. R. Munkres, Topology, Prentice-Hall of India, New Delhi, 2005.

[8] G. Pilz, Near-Rings, North-Holland, Amsterdam, 1983.

[9] K. Samei, The zero-divisor graph of a reduced ring, J. Pure Appl. Algebra 209 (2007), no. 3, 813-821.

[10] S. H. Sun, Noncommutative rings in which every prime ideal is contained in a unique maximal ideal, J. Pure Appl. Algebra 76 (1991), no. 2, 179-192.

P. DHEENA

Department of Mathematics

ANNAMAlai UNiVERSiTy

AnNAMALAINAGAR - 608 002, INDiA

E-mail address: dheenap@yahoo.com

C. Jenila

Department of Mathematics

AnNAMAlai University

AnNAMALAINAGAR - 608 002, India

E-mail address: jenincc@gmail.com 